\title{
NIVEL DE SATISFACCIÓN LABORAL EN ENFERMERAS DE HOSPITALES PÚBLICOSY PRIVADOS DE LA PROVINCIA DE CONCEPCIÓN, CHILE
}

\author{
NURSE'SJOB SATISFACTION IN PUBLIC AND PRIVATE HOSPITAL OF \\ PROVINCE OF CONCEPCION, CHILE
}

\author{
BEATRIZ FERNÁNDEZ LARRAGUIBEL* y TATIANA PARAVIC KLIJN**
}

\begin{abstract}
RESUMEN
Investigación de tipo descriptivo y correlacional que tuvo como objetivo principal determinar el nivel de satisfacción laboral de las enfermeras de centros hospitalarios públicos y privados. Se planteó que las enfermeras de los servicios privados presentaban mayor nivel de satisfacción laboral que las de los servicios públicos, y que existían diferencias significativas en el nivel de satisfacción laboral de las enfermeras, según el lugar de postulación de Enfermería al ingreso a la universidad. Se trabajó con una muestra total de 248 enfermeras hospitalarias. Se utilizaron los siguientes instrumentos recolectores: Índice de Descripción del Trabajo de Smith et al.; subescala de condiciones del trabajo de Bolda; además de preguntas relacionadas con las variables sociodemográficas y con la satisfacción en su vida personal, entre otras. Algunos de sus resultados más rel evantesfueron: las enfermeras hospital arias se encuentran sólo levemente satisfechas en su trabajo, indicando mayor satisfacción las enfermeras de los hospitales privados. Las condiciones físicas del trabajo se destacan por ser un factor deinsatisfacción para las enfermeras hospital arias, principal mente para las del sector público. Los factores remuneraciones, promociones y/o ascensos son aquellos con los que se encuentran más insatisfechas las enfermeras de ambos grupos. La interacción con sus pares, su supervisor y las actividades que realizan son aquellosfactores con los cuales obtienen mayor satisfacción. Se encontró, además, queel nivel de satisfacción laboral de las enfermerasno guarda relación con el lugar de postulación a Enfermería al ingresar a la universidad.
\end{abstract}

Palabras claves: Satisfacción laboral, enfermeras, hospitales públicos y privados.

\begin{abstract}
This research is a descriptive, and correlational one. The main purpose was to establish the level of job satisfaction in the case of those nurses working at both public and private Hospital Centers in the Province of Concepción (Chile). Such research has stated that nurses who work at private medical centers have a higher level of job satisfaction than those at public centers. Besi des, there is al so a differential among nurses' labor satisfaction level itself that has also been hypothetically claimed. The latter depends on what the program of nursing's choice was - within a numbered selection list - when applying for it. A 248-nurses-sampling was chosen. The following tools for data collecting were used, the Index of Job D escription of Smith et. al.; Bolda's sub-scale; dealing with working conditions was al so considered. The following were some of the most outstanding results: Hospital nurses show just a slight satisfaction regarding their jobs, while meaningful differences can be found between nurses working in the public and private area. With respect to the latter, nurses working in private hospital show a higher satisfaction. Physical working conditions stand out as a dissatisfaction factor in the case of hospital nurses, specially those working for the public area. Issues dealing with salaries and promotions are the ones that demonstrate greatest dissatisfaction both in private and public nurses. Interaction factors with their coworkers, supervisors, as well as with the activity they perform are the ones showing the highest satisfaction. Nurses' labor satisfaction level was found to have no relation with what the position of their choice was by the time they applied for the nursing program at the university.
\end{abstract}

Keywords: Job satisfaction, nurses, public and private hospitals.

Recepcionado: 07.05.2002. Aceptado: 28.12.2003.

*Enfermera Magíster en Enfermería mención Salud Comunitaria, Docente Universidad San Sebastián, Concepción, Chile. E-mail: bfernandez@uss.cl

**Enfermera, Profesor guía detesis, D octora en Enfermería, M agíster en Enfermería en Salud Comunitaria, Profesor Titular, Universidad deConcepción, Chile. E-mail: tparavic@udec.cl 


\section{INTRODUCCIÓN}

El concepto, la utilidad y función del trabajo han sido objeto de grandes controversias a lo largo de la historia. En la mayor parte de las sociedades la generalidad delas personas debe adecuarse de alguna manera a la exigencia de trabajar, por lo que las personas pasan más de la mitad de su tiempo de vigilia, durante las dosterceras partedesu vida, desempeñando algún tipo de trabajo.

Al mismo tiempo, el trabajo es una actividad de vastos alcances que se refleja e influye sobre casi todos los aspectos de la conducta humana, por lo que los individuos al desempeñar un trabajo no sólo trasladan a él sus habilidades intelectuales y motrices, sino también sus individualidades.

Por otra parte, el trabajo le proporciona al individuo la sensación de pertenencia a un sector de la sociedad que se considera importante, necesario y valioso. Para un individuo trabajar significa tener un propósito, expresarse y sentirse satisfecho de contribuir a la sociedad (Robbins, S., 1987; M ilcovich, T., 1994).

A la luz delos conocimientosactuales, existe un interés creciente entre los administradores, losempleados y el gobierno, por mejorar la calidad de vida en el trabajo. Existe consenso dela necesidad deestudiar al trabajador en relación con su medio ambiente laboral en el cual está inserto, ya que cualquier variación en el conjunto integrado por el hombre, la organización y el medio ambiente, afectará forzosamente a los demás. De ahí deriva la necesidad deinvestigar cientos defenómenos que ocurren a diario dentro de la organización, y uno de ellos es la satisfacción laboral, ya que extensos y variados estudios han indicado una serie de relaciones que este fenómeno tiene con la calidad de vida del trabajador, con la organización en sí y, como efecto final, con la sociedad. Existe evidencia clara que un empleado insatisfecho tiende a sufrir todo tipo de problemas de salud, tales como: cefaleas, problemas de salud mental, cardiopatías y accidentes vasculares cerebrales, entre otros (Locke, E., 1976; Stein et al., 1993; Robbins, S., 1987; Gibson, J. et al., 1985; Spector, P., 1997). Estos problemas provocarán ausentismo, rotación de personal, gastos médicos para el trabajador y costos para la empresa, los cuales van en desmedro de la organización. Como contrapartida, los estudios demuestran que la satisfacción laboral constituye un buen predictor de longevidad, de adhesión a la empresa, mejora la vida no sólo dentro del lugar detrabajo, sino quetambién influye en el entorno familiar y social. Estos aspectos son especialmente relevantes en los profesionales del área de la salud y en especial de las enfermeras, pues ellas deben cuidar la salud física y mental de quienes están a su cargo, y entregar una atención de buena calidad. Para ello deben estar bien y satisfechas en su trabajo.

El trabajo de las enfermeras y el ambiente donde lo desarrollan pareciera no ser el más propicio para derivar en satisfacción laboral. El cansancio, las escasas posibilidades de capacitación debido al sistema de turnos y la escasez de personal traen como consecuencia fatiga y desmoralización entreestas profesionales. El deber de realizar muchas tareas cuya complejidad es inferior a su formación y que comprometen su tiempo, le impiden concretar su función fundamental, que es junto al paciente en la entrega deatención y cuidados de éste (Sonis, A., 1976). Por otro lado, los turnos le dificultan la vida matrimonial y familiar, ya queéstos no coinciden con las rutinas normales de trabajo y estudios del resto de las personas, por lo que la enfermera requiere de muchos esfuerzos para compatibilizar sus actividades con las desu familia. Ade más, las bajas remuneraciones que a veces la obliga a desempeñar más de un trabajo - te niendo queasumir largas jornadas con el consiguiente desgaste que ello significa- agravan la situación. Toda esta problemática trae inevitablemente consecuencias negativas, tanto para la vida personal, laboral y tal vez para los pacientes, que repercuten finalmente en 
la sociedad dondevivimos(Thereau, J., 1987;

Pearson \& Chong, 1997).

Las enfermeras trabajan en organizaciones dedicadas a prestar ayuda en la satisfacción de la necesidad de salud de las personas; de ellas, las que trabajan en el ámbito principalmente de recuperación de la salud, lo hacen diariamente en hospitales y clínicas enfrentando múltiples problemas que derivan tanto de la atención directa del paciente, como dela administración y coordinación del equipo de salud. Es así que, durante el transcurso de mi vida laboral, he podido constatar y observar en distintos centros asistenciales los problemas y dificultades a los que se ven enfrentadas las enfermeras hospitalarias de la provincia deConcepción y que podrían estar indicando que el nivel de satisfacción laboral deéstas no es el mejor. Las enfermeras resienten los conflictos que se generan dentro de los equipos detrabajo, las dificultades con sus jefes directos, con sus pares y con el personal a su cargo, ya que éstas entorpecen la comunicación, alteran la armonía y el desarrollo del trabajo, tornándolo dificultoso y poco gratificante. Las enfermeras se molestan por el escaso espacio, muy reducido 0 a veces inexistente, asignado en sus lugares de trabajo, lo que ellas perciben como falta de reconocimiento hacia su grupo profesional de parte de la institución en que se desempeñan. Por otra parte, sus históricas bajas remuneraciones y los escasos ascensos son dificultades que, junto a otras, estas profesionales deben enfrentar, con el consiguiente malestar que les implica.

Es importante destacar que estas quejas y dificultades las han manifestado tanto las enfermeras dehospitales públicos como de hospitales privados, con énfasis en diferentes aspectos dependiendo del tipo de hospital que se trate.

Por todo lo anteriormente expuesto, surge la siguiente pregunta: ¿Cuál es el nivel de satisfacción laboral de las enfermeras de hospitales públicos y privados de la provincia de Concepción?

\section{TIPO DE INVESTIGACIÓN}

La presenteinvestigación es un estudio detipo descriptivo, correlacional.

\section{PROPÓSITO DEL ESTUDIO}

El propósito deesteestudio fue determinar el nivel de satisfacción laboral de las enfermeras de los hospitales públicos y privados de la provincia de Concepción, Chile, e identificar algunos factores relacionados con esta satisfacción.

\section{OBJETIVOS ESPECIFICOS}

- Caracterizar el perfil de las enfermeras, de los hospitales públicos y privados de la provincia, según las variables sociodemográficas.

- Conocer el nivel de satisfacción laboral de las enfermeras, de los hospitales públicos y privados de la provincia, en relación a las variables sociodemográficas

- Comparar el nivel de satisfacción laboral de las enfermeras del sector público con el nivel de satisfacción de las del sector privado.

- Conocer el nivel de satisfacción laboral de las enfermeras de los hospitales públicos y privados de la provincia, en relación a las condiciones físicas de su trabajo.

- Conocer el nivel de satisfacción laboral respecto a los diversos factores del constructo trabajo en ambos grupos de enfermeras.

- Conocer el nivel de satisfacción de las enfermeras de los hospitales públicos y privados, en relación a su vida personal.

- Relacionar el nivel de satisfacción en la vida personal con el nivel de satisfacción laboral general.

- Conocer si existe relación entre el nivel de satisfacción general y el lugar de opción en la elección de la carrera de Enfermería, al momento del ingreso a la universidad. 


\section{HIPÓTESIS}

- Existen diferencias significativas en los niveles promedio de satisfacción laboral, entre las enfermeras de los hospitales públicos y privados de la provincia de Concepción.

- Existen diferencias significativas en el nivel de satisfacción laboral general, según el lugar deopción al momento dela elección de la carrera deEnfermería, al ingreso a la universidad, entre las enfermeras de los hospitales públicos y privados de la provincia de Concepción.

Para esta investigación se tuvo como marco referencial teórico las conceptualizaciones de Edwin Locke, psicólogo laboral.

El modelo conceptual de Lockese basa en dos corrientes teóricas de explicación de la motivación conductual del hombre. La primera se refiere a las teorías Ilamadas de proceso, desde las cuales la satisfacción laboral puede ser vista como el estado resultante de la percepción quetenga el individuo de su trabajo como herramienta, quelepermitaalcanzar o lograr un importante valor a condición de que sus valores sean compatibles con sus necesidades y vistas como esenciales para el bienestar del individuo. Entre éstas tenemos la Teoría de las Expectativas y la Teoría de la Equidad. La segunda corriente de teorías se refiere a las teorías de contenido, y desde este punto de vista estas teorías intentan explicar qué necesidades 0 valores deben ser satisfechos por el individuo para estar satisfecho con su trabajo. Aquí tenemos la Teoría de las $\mathrm{Ne}$ cesidades Jerarquizadas de Maslow y la de Higieney M otivación de Herzberg (Robbins, S., 1987; Chiavenato, Y., 1998; Dunnette, M., 1979; Gibson, J. y col., 1985). Locke, en basea la revisión deestas teorías, elabora su conceptualización y manifiesta que el individuo logra satisfacción laboral al apreciar que el trabajo le facilita la realización de valores importantes para él y que éstos son compatibles con sus necesidades. Es así que el individuo hace una evaluación de los hechos, obje- tos o situaciones que derivan del trabajo y según sus expectativas, sus necesidades tanto físicas como sicológicas, realiza un juicio interno de valor, el cual puede ser positivo o negativo para el individuo, por lo que tendrá una reacción emocional placentera o displacentera al respecto y en consecuencia obtendrá satisfacción o insatisfacción.

Este autor destaca la importancia de los valores del individuo, de sus necesidades, de sus expectativas, de sus percepciones y de sus juicios, especificando con su modelo causal cómo estas variables se combinan para determinar el grado de satisfacción laboral. Además, señala que para comprender las actitudes hacia el trabajo es necesario analizar los distintos factores del mismo, algunos de los cuales se estudiaron en esta investigación, tales como: las actividades propiamente tales, las remuneraciones, las condiciones físicas del trabajo, las promociones y/o ascensos, las políticas de la empresa, las relaciones con el supervisor o jefey las interacciones con los compañeros de trabajo.

Se han realizado múltiples investigaciones respecto a la satisfacción laboral, pero sólo en las últimas décadas se han preocupado por la satisfacción de las enfermeras. Es así como diversos autores han encontrado los siguientes resultados: Kramer y Schmalenberg (1992) en EE.UU. observaron que la autonomía, el estilo de gestión y el reconocimiento por partedela institución dondetrabajaban, influían de forma importante en la satisfacción laboral de estas enfermeras. Salinas y col. (1994), en M éxico, informaron que las enfermeras participantes en su estudio manifestaron satisfacción en todos los aspectos del trabajo a excepción del clima y la armonía intragrupo. Chávez y col. (1996), en M éxico, indicaron que las enfermeras participantes en su investigación mostraban un nivel moderado desatisfacción laboral. M oss y Rowler (1997), en EE.UU., mostraron queel estilo participativo y cercano de dirección mejoraba la satisfacción laboral de las enfermeras. Krichkaew (1998), en Tailandia, encontró el mismo re- 
sultado. En nuestro país, Paravic (1998), Parra (2002) y Zúñiga (2002) pudieron constatar en sus respectivas investigaciones que el nivel de satisfacción laboral de las enfermeras era leve, encontrando gran insatisfacción en sus remuneraciones y en las políticas de promoción y ascenso de sus instituciones, hallazgos estosúltimos recurrentes en los distintos estudios relacionados con la satisfacción laboral delas enfermeras.

\section{SUJETOSY MÉTODOS}

La población la conformaron las enfermeras de todos los centros hospitalarios, tanto públicos como privados, de la provincia deConcepción, Chile, con un total de 447 enfermeras. En esta investigación se trabajó con el 50\% delas enfermeras del sector público yel 100\% de las del sector privado. Se excluyeron dos centros hospitalarios que participaron en la prueba piloto (uno público y otro privado), cada uno con seis enfermeras. Por lo que la muestra quedó constituida por 248 enfermeras hospitalarias, 147 del sector público y 101 enfermeras del sector privado.

Para la realización de este estudio se solicitó autorización a directores de Servicios de Salud de Concepción y Talcahuano, como también a los diferentes directores delos hospitales privados que se integraron en el estudio.

Los instrumentos fueron aplicados personalmente por la autora, en el lugar de trabajo de cada una de las enfermeras, en forma grupal por servicios, o por grupos afines en los diferentes hospitalestanto públicos como privados. El tiempo promedio de las respuestas a las encuestas fue de 12 minutos. Se garantizó el anonimato y confidencialidad a las enfermeras participantes, con el sellado inmediato de cada encuesta.

Para la recolección de la información se utilizaron los siguientes instrumentos: una encuesta elaborada por la autora, relacionada con los datos biosociodemográficos de las enfermeras; la subescala de condiciones físicas del trabajo de Bolda (1958), y el Índice de Descripción del Trabajo de Smith et al. (1969), el cual se componede cinco subescalas, las que se refieren a las actitudes del trabajador frentea las actividades querealiza, interacción con los compañeros del trabajo, su jefe, las remuneraciones y los ascensos y/o promociones. Por último, se utilizó una escala análoga visual, con la que se midió el nivel de satisfacción laboral general y la satisfacción con la vida personal, además de una pregunta cerrada, relacionada con el lugar de postulación que ocupó Enfermería al momento de ingresar a la universidad.

Las escalas utilizadas en el presente estudio fueron validadas por diversos autores en estudios realizados en distintos países, tanto en su contenido como en su constructo.

En cuanto a su confiabilidad, se utilizó el Alfa deCronbach, quedemostró una alta confiabilidad.

Los datos fueron procesados en el Departamento deEstadística de la Facultad deFísica yM atemática delaUniversidad deConcepción. Para el análisis de los datos se dividieron en dos grupos: las enfermeras de los hospitales públicos y las enfermeras de los hospitales privados. Se utilizaron tablas de frecuencia, porcentajes, promedios, desviación estándar, t deStudent, razón $\mathrm{F}$ y coeficiente de correlación $r$ de Pearson.

\section{RESULTAD OS, DISCUSIÓN Y CONCLUSIONES}

El perfil biosociodemográfico delas enfermeras de los hospitales públicos se caracteriza porquela mayoría delas enfermeras tienemás de 41 años, tienen hasta 2 hijos, y tienen pareja. Un porcentaje importante trabaja más de 20 años en la institución, y sus ingresos mensuales brutos son inferiores a $\$ 450.000$ (año 2000), a diferencia del perfil biosociodemográfico de las enfermeras de los hospitales privados, dondela gran mayoría de ellas 
tiene entre 23 y 40 años, hasta 2 hijos, y con pareja y, mayoritariamente, trabajan menos de 19 años en la institución, y sus ingresos mensual es brutos son igualmentebajosquelos de las enfermeras de los hospitales públicos.

En general, las enfermeras se perciben levementesatisfechas con su trabajo, resultado que concuerda con los estudios de Zúñiga, Y. (2002) y Parra, S.(2002) sobre enfermeras de atención primaria y profesionales deenferme ría de los SAMU, respectivamente. Se observó también que las enfermeras de los hospitales privados se encontraban más satisfechas que las de los hospitales públicos, resultado que hace aceptar la hipótesis alternativa que plantea este estudio: que existen diferencias significativas entre los niveles promedio de satisfacción laboral de las enfermeras de los hospitales públicosy privados de esta provincia.

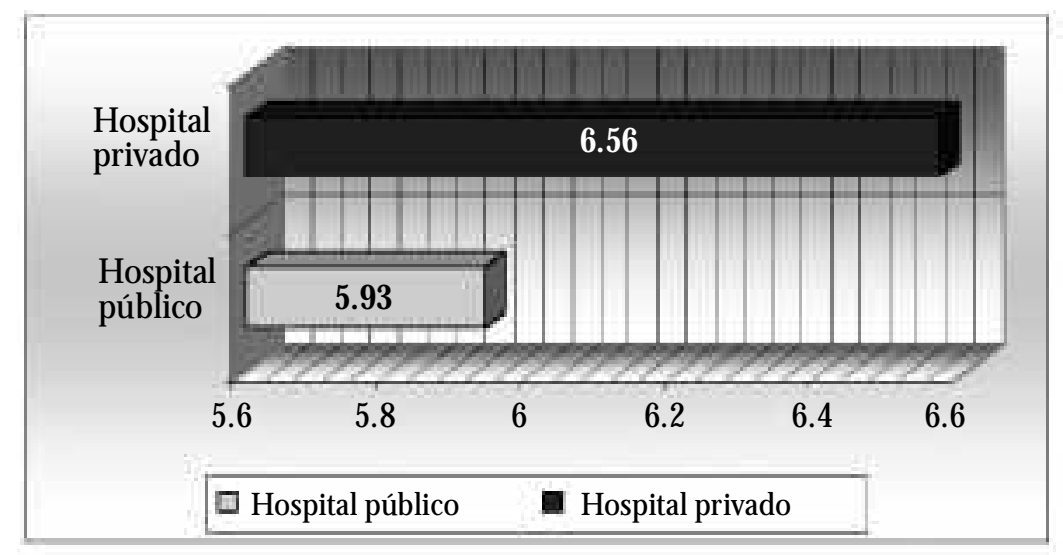

Gráfico $\mathbf{N}^{\circ} \mathbf{1}$. Nivel de satisfacción laboral general enfermeras de los hospital es públicos y privados dela provincia de Concepción, según escala análoga visual.

Fuente: Investigación Nivel de Satisfacción Laboral de las Enfermeras de los H ospitales Públicos y Privados de la Provincia de Concepción VIII Región, Chile, 2001.

Al observar la relación entre las variables sociodemográficas y el nivel de satisfacción laboral general de las enfermeras hospitalarias, no se encontraron grandes diferencias dentro de los grupos de enfermeras de hospitales públicos y privados. Las enfermeras que se encontraban más satisfechas en ambos tipos de hospitales son aquellas que tienen entre 41 y 58 años, con 3 o más hijos, su ingreso mensual bruto esmayor de $\$ 600.000$. Sin embargo, difieren en que se encuentran más satisfechas las de la categoría separadas deloshospitales públicos, y quetienen 5 años o menos de trabajo en la institución, a dife- rencia de las enfermeras de los hospitales privados, en los cual es las más satisfechas corresponden a la categoría casadas, y con 20 años y más de trabajo.

Por otra parte y en relación al servicio en el cual trabajan, las que se encuentran más satisfechas son las enfermeras de los hospitales privados que pertenecen a aquellos servicios agrupados en las categorías D (urgencia adultos, servicio deU CI y UTI), A (medicina, gastroenterología, oncología, diálisis, urología, cardiología, dermatología, salud ocupacional y unidad de toma de muestras) y $B$, respectivamente(cirugía, neurocirugía, pabe- 
Ilón, post-operatorio, traumatología, unidad de quemados, y otorrino), a diferencia de las enfermeras de los hospitales públicos, donde las más satisfechas pertenecen a la categoría $\mathrm{F}$ (coordinadoras generalesy enfermeras deinfeccionesintrahospitalarias) y las enfermeras de los servicios de las categorías A y D.

Respecto a la satisfacción con las condiciones físicas del trabajo, las enfermeras de hos- pitales privados están más satisfechas que las enfermeras de los hospitales públicos en este aspecto, y presentan diferencias significativas y altamente significativas en casi la totalidad de los ítemes de la subescala aplicada. Ellas identifican su lugar detrabajo como riesgoso einadecuado, el queesademás asociado a falta de preocupación por su salud y seguridad de parte de la institución donde laboran.

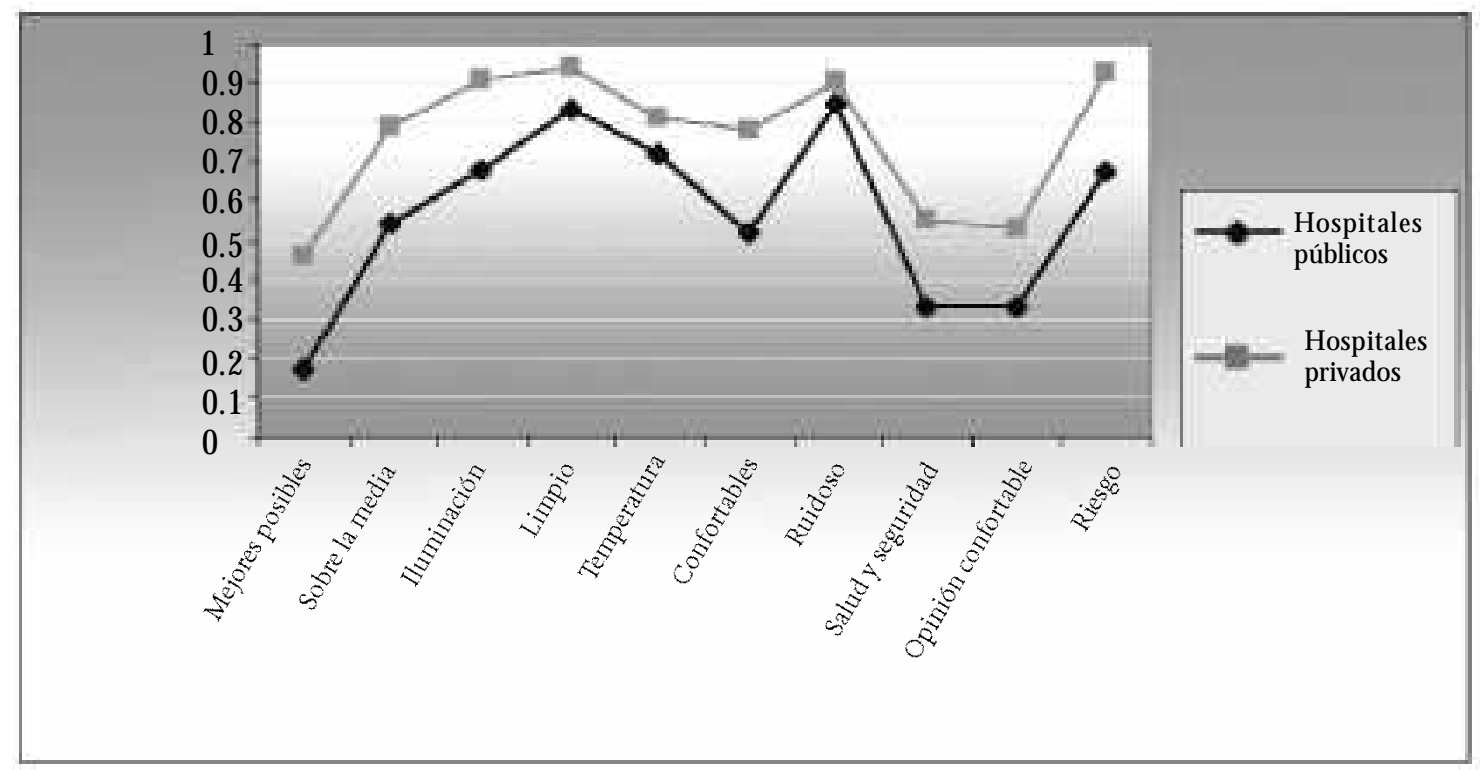

Gráfico $\mathbf{N}^{\circ}$ 2. Nivel de sati sfacción laboral con las condiciones físicas del trabajo de las enfermeras de hospitales públicos y privados de la provincia de Concepción, según los diversos ítemes de las subescalas de Bolda. Fuente: Idem Gráfico ${ }^{\circ} 1$.

Las condiciones donde se realiza el trabajo, según la literatura revisada, influye en la satisfacción laboral de las personas, y especialmente sensibles a ellas son las mujeres (Locke, E. , 1976; Robbins, S., 1987; Sonis, A., 1987; Escuela Andaluza de Salud Pública de España, 1997; Valenzuela, S. y Do Carmo, 2000).

Avendaño y col. (1997) señalan que dentro de las características delas condiciones de trabajo de las enfermeras se encuentran las siguientes: altas cargas físicas, exposición constantea contaminantesfísicos, químicos y biológicos, como también a accidentes. Por otra parte, Salvo (1996) encontró que el ruido, la iluminación, el aseo y ventilación deficiente, la temperatura inadecuada y los malos olores, sumado al tener quetrabajar con equipos y materiales deficientes, era lo que más molestaba y tensionaba a las enfermeras.

En relación al nivel de satisfacción laboral de las enfermeras, respecto a cada uno de los factores del trabajo, del IndicedeD escripción del Trabajo de Smith et al., ellas presentan un nivel de satisfacción sobreel punto neutro en las subescalas actividades, supervisor 0 jefe y grupo con quien interactúan más cercanamente, lo que indica que en estos tres aspec- 
tos las enfermeras se encuentran con una leve tendencia a la satisfacción. Sin embargo, respecto a la subescala promociones $y / 0$ ascensos, ellas se encuentran considerablemente bajo el punto neutro, lo quesignifica quepresentan una clara tendenciaa la insatisfacción, estando las enfermeras de los hospitales privados menos insatisfechas que las de los hospitales públicos. Por otra parte, el factor promociones y/o ascensos se observa como un factor muy influyente de insatisfacción laboral para ambos grupos de enfermeras, ya que son percibidas como injustas y poco transparentes dentro de las instituciones hospitalarias. Paravic, T. (1998) y Parra, S. (2002) también encontraron que las promociones y/o ascensos eran uno de los aspectos donde se encontraban más insatiffechas las enfermeras participantes en sus respectivos estudios. Robbins, S. (1987) señala que, si en la opinión de los empleados la compensación y los estímulos son justos y racionales, aumentará el nivel de satisfacción, y, si son injustos, los empleados tendrán unaaltainsatisfacción al respecto, al igual que en el presente caso.

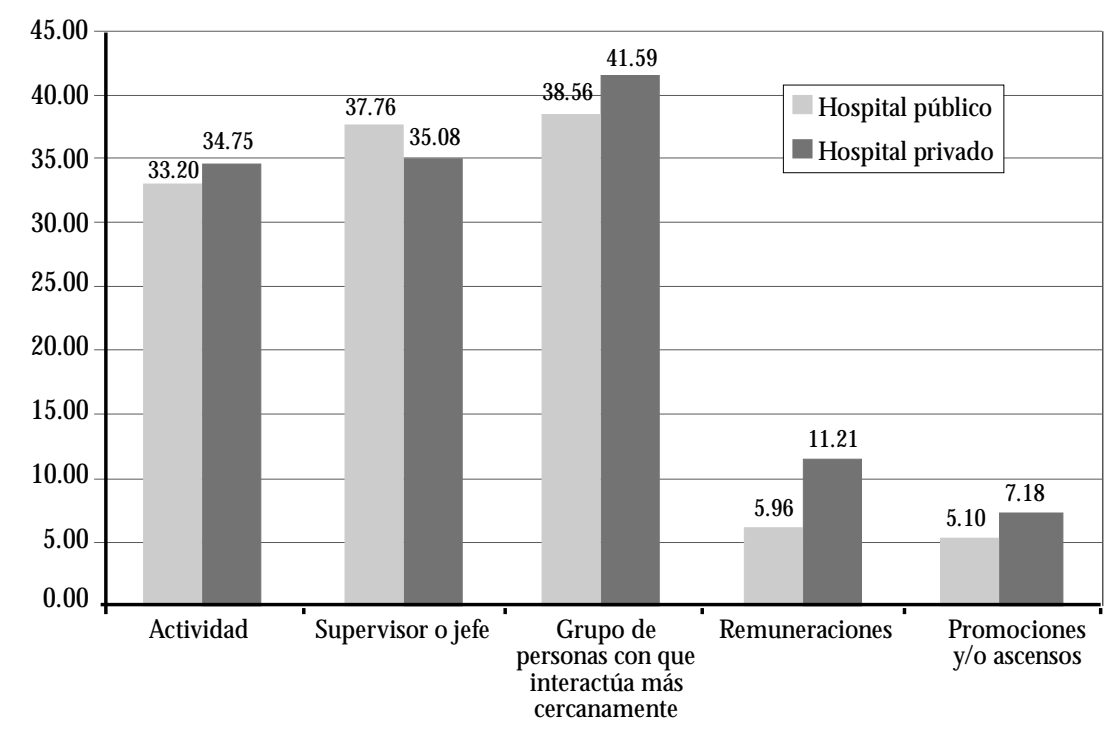

Gráfico $\mathbf{N}^{\circ}$ 3. Nivel de satisfacción laboral con las condiciones físicas del trabajo de las enfermeras de hospitales públicos y privados de la provincia de Concepción, según las diferentes subescalas del Índice de Descripción del Trabajo de Smith et al.

Fuente: Idem Gráfico $N^{\circ} 1$.

Otro factor deinsatisfacción en ambosgrupos de enfermeras son las remuneraciones, sobre las cuales ellas manifiestan claramente su descontento, aunque más marcadamente en las enfermeras de los hospitales públicos, quienes presentan diferencias altamente significativas en este aspecto con las enfermeras de los hospitales privados. Ambos grupos de enfermeras consideran sus remuneraciones malas, injustas y menores de lo que merecen. Parra, S. (2002) y Zúñiga, Y. (2002), Avendaño y col. (1997); y Salvo, C. (1996) encontraron los mismos resultados en los profesionales de enfermería en sus respectivas investigaciones.

En relación a esta problemática, Locke, $\mathrm{E}$. (1976) manifiesta que el dinero es valorado como un símbolo de realización, de reconocimiento, de status o como forma de obtener otros valores, como seguridad o libertad de acción, además de ser un punto de referencia por medio del cual los empleados comparan su valor con otros. Por otra parte, Robbins, S. 
(1987) señala que las personas desean sistemas de sueldos justos, definidos y acordes a sus expectativas. Cuando el pago se visualiza como justo a partir delas demandas del puesto, el nivel de habilidades individuales, y los niveles de sueldo de la comunidad, es posible que haya satisfacción.

Cabedestacar queen laúnica subescala que las enfermeras de hospitales públicos logran un mayor nivel de satisfacción que las enfermeras de los hospitales privados, es en la que se refiere al supervisor o jefe. Según la experiencia de la autora, esto se debe a la mayor verticalidad y menor espacio de poder de las enfermeras de los hospitales privados. Krichkaew en Tailandia (1998) encontró que los estilos participativos de dirección o supervisión hacen que las enfermeras tengan un nivel más alto de satisfacción laboral, lo que finalmente llevaría a entregar un servicio más efectivo. Por otra parteM oss\& Rowler (1997) también encontraron que el estilo cercano de dirección y el participativo mejoraba la satisfacción laboral de las enfermeras.

En relación al nivel de satisfacción en la vida privada de las enfermeras, se encontró que ellas estaban más satisfechas en este ámbito que en el aspecto laboral.

En cuanto al nivel de satisfacción laboral y su relación con el lugar deopción al momento de la postulación a la carrera de Enfermería, se encontró que cerca del $61 \%$ de las enfermeras postuló en primer lugar a la carrera, no encontrándose diferencias significativas en el nivel de satisfacción laboral de estas enfermeras, respecto a aquellas que postularon en segunda o tercera opción. Dado este resultado, se rechazó la segunda hi pótesis alternativa de este estudio, que dice que existen diferencias significativas en el nivel desatisfacción laboral de las enfermeras según lugar de postulación a la carrera de Enfermería como primera opción al ingreso a la universidad, de aquellas que postularon en segunda o tercera opción.

Esta investigación es parte de un proyecto mayor sobresatisfacción laboral en Enferme- ría y que se complementa con otros estudios sobre el tema, tales como los realizados en enfermeras académicas, en servicios de atención prehospitalaria y de atención primaria, en esta región y en otras. Y tiene como finalidad conocer la realidad nacional formulando así un diagnóstico quepermita proponer a las autoridades de los distintos centros e instituciones de salud la implementación de programas que ayuden a mejorar u optimizar los factores del trabajo que se identifiquen como influyentes en la satisfacción laboral de las enfermeras de nuestro país, y lograr, así, que éstas trabajen en ambientes laborales más confortables, seguros y agradables, con políticas deremuneraciones y depromocionesy ascensos justas y equitativas, todo lo cual influirá en la satisfacción laboral de la enfermera, en su vida personal, en la institución donde laboran y finalmente en el paciente o usuario de la atención de salud.

\section{REFERENCIAS BIBLIOGRÁFICAS}

AVEN DAÑ O, C.; Grau, P.; Yus, P. (1995). Riesgos para la salud de las enfermeras del sector público en Chile. Revista Enfermería. Colegio de Enfermeras de Chile. Dic. 15-26.

BOLDA (1984). Escala opinión sobre el trabajo. 1958 in Belli,V. y Dela Coleta. Adaptaçao e padronização de escalas de atitudes para situaçoes de trabalho. Partell. Conselho Nacionalde de desenvolbimento cientifico e tecnológico. Brasil.

CHÄVEZ y col. (1996). Satisfacción laboral en médicos y enfermeras. Revista M édica IM SS. M ayo-junio, 34(3) :253-256. M éxico.

CHIAVENATO, Y. (1990). Administracion de recursos humanos. Trad. M aría Isabel Fittipaldi de Ruíz. M éxico, M cGraw-Hill.

DUN NETTE, M . y Kirchner, W. (1979). Psicología industrial. Edit. Trillas. M éxico.

ESCUELA Andaluza de Salud Pública (EASP) (1997). Experiencia del área hospitalaria, Virgen M acarena de Sevilla. La satisfacción del profesional, termómetro dela organizacional. Diario médico gestión. Septiembre.

GIBSON, J. et al. (1985). Organizaciones, conducta, estructura y proceso. Edit. Interamericana, M éxico.

KRAM ER, M . \& Schmalenberg Cl. (1992). Trabajo, satisfacción y retención, reflexiones para la decada 
delosnoventa. Nursing. Enero-febrero. Barcelona, España.

KRICHKAEW, K. (1998). Head nurse management style and staff nurse job satisfaction in Northerm Regional CentreH ospitals. Chiang Mai, Universidad Regional de Tailandia. Tailandia. http://www. chiangmai .ac.th/abstract1998/Abstract/nur/abstract/nur980206.html.

LOCKE, E. (1976). De nature and causes of job satisfaction, in Dunnette, M. Handbook of industrial and organizational psychology, Rand-Mc Nally College, p. 1297-1349. Chicago. EE.UU.

MILKOVICH, T., Boudreau W. (1994). Dirección y administración de recursos humanos, un enfoque estratégico. Edit. Addison Wesley I beroamericana, EE.UU.

M OSS, R. \& Rowler, C. (1997). Staff nurse job satisfaction and management style. Nursing management. Jan. 28(1): 32-34. EE.UU.

PARAVIC,T. (1998). Satisfacción laboral de mujeres académicas dela Universidad deConcepción. Chile. Tesis para optar al grado de Doctor en Enfermería Universidad de Sao Paulo. Brasil.

PARRA, S. (2002). Satisfacción laboral de las(os) enfermeras(os) quetrabajan en el servicio deatención médica de urgencia (SAMU) en la Región M etropolitana y VIII Región de Chile. Tesis para optar al grado de magíster en Enfermería Comunitaria, Universidad de Concepción, Chile.

PEARSON, A. \& Chong, J. (1997). Contributions of job content and social information on organizational commitment and job satisfaction: an exploration in a Malaysian nursing context. Journal of Ocupational Psychology, 70:357-374.

ROBBINS, S. (1987). Comportamiento organizacional. Conceptos, controversias y aplicaciones, 3a edición, Edit. Prentice-H all H ispanoamericana S.A. M éxico.
SALINAS, O. C. y col. (1994). La satisfacción laboral y su papel en la evaluación de la calidad de la atención médica. Salud Pública, 36(1):22-29, M éxico.

SALVO, C. (1995). Estrés laboral, extralaboral y síntomas psicosomáticos en la mujer enfermera. Tesis para optar al grado de magister en Enfermería M édico-Quirúrgica, Universidad de Concepción, Servicio de Salud VI Región, Chile.

SONIS, A. y col. (1976). Atención de salud, tomo II, Medicina sanitaria y administración de salud, Zera Ed., Edit. El Ateneo, Chile.

SM ITH, D. C.; Kendall, L. M. \& Hulin, C. L. (1969). The measurement of satisfaction in work and retirement, Chicago, Rand Mc Nally.

SPECTOR, P. (1997). Job satisfaction aplication, assesment, cause and consequences. EE.UU. Edit. Sage publication.

STEIN, A. J. et al. (1993). Consequences of adolescent drug use on young adult job behavoir and job satisfaction. J ournal of Applied Psychology, 78(3): 463-474.

THEREAU, J. (1987). El estrés físico y sicológico en el trabajo, Editorial Centro de Publicaciones, M inisterio del Trabajo y Seguridad Social, M adrid, España.

VALENZUELA, S. y Do Carmo, M . (2000). Accidentes del trabajo: La situación de la mujer del equipo de enfermería en dos hospitales clínicos chilenos, Ciencia y Enfermería. Rev. Iberoamericana de Investigación, Vol. VI, 1, Depto. de Enfermería Universidad de Concepción, Chile, pp. 67-78.

ZUÑ IGA, Y. (2002). Nivel de satisfacción laboral de las enfermeras de consultorios urbanos y rurales de las comunas de la provincia de Concepción. Avance de tesis para optar al grado de M agíster en Enfermería mención Salud Comunitaria, Universidad de Concepción, Chile. 